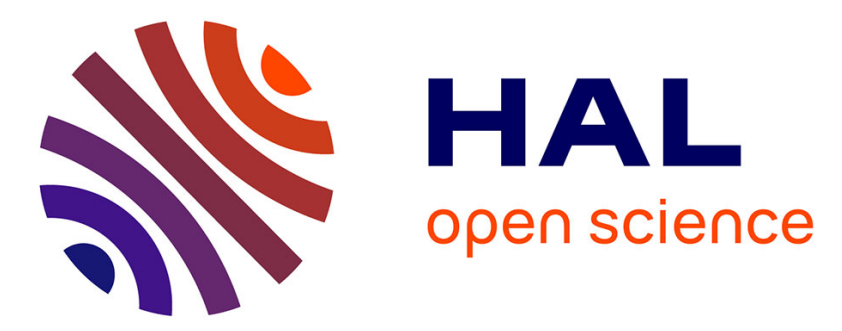

\title{
Influence of two atmospheric transport models on inferring sources and sinks of atmospheric $\mathrm{CO} 2$
}

P. Bousquet, P. Ciais, P Monfray, Yves Balkanski, Michel Ramonet, P. Tans

\section{To cite this version:}

P. Bousquet, P. Ciais, P Monfray, Yves Balkanski, Michel Ramonet, et al.. Influence of two atmospheric transport models on inferring sources and sinks of atmospheric CO2. Tellus B - Chemical and Physical Meteorology, 1996, 48 (4), pp.568-582. 10.3402/tellusb.v48i4.15932 . hal-02874100

\section{HAL Id: hal-02874100 https://hal.science/hal-02874100}

Submitted on 18 Jun 2020

HAL is a multi-disciplinary open access archive for the deposit and dissemination of scientific research documents, whether they are published or not. The documents may come from teaching and research institutions in France or abroad, or from public or private research centers.
L'archive ouverte pluridisciplinaire HAL, est destinée au dépôt et à la diffusion de documents scientifiques de niveau recherche, publiés ou non, émanant des établissements d'enseignement et de recherche français ou étrangers, des laboratoires publics ou privés. 


\section{Influence of two atmospheric transport models on inf erring sources and sinks of atmospheric $\mathrm{CO}_{2}$}

\section{P. Bousquet, P. Ciais, P. Monfray, Y. Balkanski, M. Ramonet \& P. Tans}

To cite this article: P. Bousquet, P. Ciais, P. Monfray, Y. Balkanski, M. Ramonet \& P. Tans (1996) Influence of two atmospheric transport models on inf erring sources and sinks of atmospheric $\mathrm{CO}_{2}$, Tellus B: Chemical and Physical Meteorology, 48:4, 568-582, DOI: 10.3402/tellusb.v48i4.15932

To link to this article: https://doi.org/10.3402/tellusb.v48i4.15932

$$
\begin{aligned}
& \text { (c) } 1996 \text { The Author(s). Published by Taylor \& } \\
& \text { Francis. }
\end{aligned}
$$

\section{曲 Published online: 18 Jan 2017.}

Submit your article to this journal

\section{Щ Article views: 23}

Q View related articles $\widetilde{ }$

47 Citing articles: 4 View citing articles 둔




\title{
Influence of two atmospheric transport models on inferring sources and sinks of atmospheric $\mathrm{CO}_{2}$
}

\author{
By P. BOUSQUET*1, P. CIAIS ${ }^{2}$, P. MONFRAY ${ }^{1}$, Y. BALKANSKI ${ }^{2}$, M. RAMONET ${ }^{1,3}$ and \\ P. TANS ${ }^{3}$, ${ }^{1}$ Centre des Faibles Radioactivités (CFR), CNRS, 1 Av. de la Terrasse, 91198 Gif-sur-Yvette, \\ France; ${ }^{2}$ Laboratoire de Modélisation du Climat et de l'Environnement (LMCE), CEN de Saclay, Orme \\ des Merisiers, 91191 Gif-sur-Yvette, France; ${ }^{3}$ Climate Monitoring and Diagnostics Laboratory, National \\ Oceanic and Atmospheric Administration (NOAA/CMDL), 325 Broadway, Boulder, Colorado, 80303 USA
}

(Manuscript received 2 November 1995; in final form 11 April 1996)

\begin{abstract}
Atmospheric transport models are a source of uncertainty in the diagnostics of the $\mathrm{CO}_{2}$ sources and sinks. We propose here a protocol to compare two transport models: a 2-dimensional (2D) and a 3-dimensional (3D) model, based on 3 different experiments that reveal the ability of each model to account for the different components of the atmospheric carbon cycle. The $2 \mathrm{D}$ model we use is the one described by Tans et al. and the 3D model is the TM2 model, developed by Heimann et al. First, we conduct the same fossil fuel experiment in both models and show that the $2 \mathrm{D}$ model has a stronger inter-hemispheric mixing than the $3 \mathrm{D}$ model $(\sim 25 \%)$, even though the 2D model presents a weaker intra-hemispheric mixing above source regions (experiment A). The influence of year-to-year variability of transport on the latitudinal profile in fossilfuel $\mathrm{CO}_{2}$ appears to be weak for the 1990s. We then use a set of "all but fossil fuel" fluxes, originally inferred from the 2D model, as an input to the 3D model (experiment $\mathrm{B}$ ). Even if the main discrepancy on the resulting latitudinal $\mathrm{CO}_{2}$ concentrations occurs between the $2 \mathrm{D}$ and 3D models in the tropics and at the mid-northern latitudes, the differences implied by three longitudinal distributions tested in the 3D model are important and can be explained by a few global transport mechanisms. Finally, we quantify the differences in latitudinal $\mathrm{CO}_{2}$ concentrations observed in experiment $B$ in terms of net carbon fluxes at the surface. To do so, an inverse calculation of the $\mathrm{CO}_{2}$ fluxes in latitude and time is performed with the $3 \mathrm{D}$ model, using as an input a smoothed latitudinal profile of atmospheric measurements for the period 1990-1993 (experiment $C=A+B$ ). We find with the 3D model that, averaged on the period 1990-93, the equatorial release is reduced by $40 \mathrm{Tmol} \mathrm{yr}^{-1}$ (roughly $25 \%$ of the original source) compared with the initial $2 \mathrm{D}$ budget and is shifted southward by roughly $10^{\circ}$. The mid northern latitude

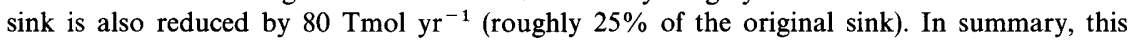
study shows that the changes in the carbon budget required when moving from the $2 \mathrm{D}$ model to this 3D model are important, but they are not radical changes.
\end{abstract}

\section{Introduction}

Carbon dioxide, the major anthropogenic greenhouse gas, is continuously exchanged between atmosphere, land and ocean. In addition, fossil fuel burning and changes in land use have intro-

\footnotetext{
* Corresponding author.email: bousque@cea.fr.
}

duced anthropogenic perturbations in the natural carbon cycle. The $\mathrm{CO}_{2}$ growth rate is of $1.5 \mathrm{ppm}$ $\mathrm{yr}^{-1}$ for the 1980 's with strong inter-annual variations (Conway et al., 1994; Francey et al., 1995; Keeling, 1995). On average, $50 \%$ of the anthropogenic source actually ends up accumulating in the atmospheric reservoir, the other half being absorbed either by the oceans or by land eco- 
systems. The residence time of $\mathrm{CO}_{2}$ is much greater in the oceans than in land ecosystems, which could imply a different long-time response of the climate system depending on which one is the main reservoir. Thus, relating the spatial distribution of $\mathrm{CO}_{2}$ as provided by the measurement network to sources and sinks is of great interest in balancing the carbon budget and devising future strategies for $\mathrm{CO}_{2}$ emissions (IPCC, 1995).

Many studies have presented the inverse process of inferring sources and sinks of $\mathrm{CO}_{2}$ from concentration data. Two major schemes have been used thus far to carry out inverse calculations from concentration fields : the synthesis inversion (Enting et al., 1995, 1993 ; Heimann and Keeling, 1989 ) and the mass balance inversion (Enting and Mansbridge, 1991; Tans et al., 1989). The synthesis inversion starts with a first guess of the spatial distribution of each source and uses a transport model to calculate the spatial distribution of the $\mathrm{CO}_{2}$ mixing ratio corresponding to each source. Then the best linear combination of sources, varying the overall strength but not spatial pattern of individual sources, is estimated so that the calculated concentration field matches the observed concentrations (Enting et al., 1993; Tans et al., 1990). Enting et al. (1993, 1995) described and improved this process by using a Bayesian technique to establish source combinations, which also takes into account independent and prior knowledge of sources with their estimated errors. The limitation of the method is the obligation to define an a priori spatial distribution of each source. In the Bayesian approach the prior "knowledge" might nudge the solution in the right direction, but also in the wrong direction if the prior knowledge is incorrect; the Bayesian inverse method is intentionally biased. In the mass-balance method, the surface concentrations of $\mathrm{CO}_{2}$ are prescribed in an atmospheric transport model and the sources and sinks are then determined so that the modelled concentration fields at the surface match the observations. Ideally, the surface concentrations are described everywhere based on observations. This method, however, assumes that the $\mathrm{CO}_{2}$ measurement network is representative of the spatial distribution of the concentrations, while in fact the number of monitoring sites is still insufficient especially in the southern hemisphere to fully determine the inversion and allow a reliable 3D inversion. This is the reason why most of the studies of this type that have been conducted until now have been based on two-dimensional transport models (Conway et al., 1994; Enting and Mansbridge, 1991; Tans et al., 1989; Enting and Mansbridge, 1989) or on a zonally uniform inversion with 3d transport (Law et al., 1992).

All these studies agree that the north-south concentration gradient implies an important sink in the northern hemisphere. Enting and Mansbridge (1991) include the cycles of methane $\left(\mathrm{CH}_{4}\right)$ and carbon monoxide $(\mathrm{CO})$ with no seasonality into the consideration of the north-south gradient, but that does not introduce much change in the above conclusion. However, the nature of this sink, whether it is oceanic or terrestrial, remains somewhat controversial (Heimann and Keeling, 1989; Tans et al., 1990), although recent work, based on $\delta^{13} \mathrm{C}$ ratios, suggests, amongst other things, that the Northern Hemisphere land biosphere did absorb large quantities of carbon over the past few years (Francey et al., 1995; Keeling, 1995; Ciais et al., 1995a,b).

Nevertheless, all such diagnostics of the global carbon budget rely to model the $\mathrm{CO}_{2}$ concentrations in the atmosphere, which raises the question of how sensitive the derived $\mathrm{CO}_{2}$ budget is to the prescribed atmospheric transport. Apart from model validation with inert tracers like ${ }^{222}$ Radon and ${ }^{85} \mathrm{Krypton}$ the only method we can use to tackle this problem is model comparison. In a first attempt to do so, (Rayner and Law, 1995, Transcom I) have run 12 transport models using the same sets of $\mathrm{CO}_{2}$ fluxes, and compared the different modelled concentration fields. This comparison shows that atmospheric transport models predict very different concentration fields starting from the same prescribed source. In the present study, we compare in detail the transport patterns of two atmospheric models and the implication of the transport differences on the carbon budget of the 1990s. These models are both widely used in modelling atmospheric $\mathrm{CO}_{2}$ (see Table 1). The first model is a bidimensional model (latitude-vertical) first developed by Plumb and Mahlman (1987) and further adapted by Tans et al. (1989) at the Climate Monitoring and Diagnostics Laboratory of the National Oceanic and Atmospheric Administration (NOAA/CMDL). It is used to determine sources and sinks of $\mathrm{CO} 2$ from the measurements of the NOAA/CMDL network, through an "inverse" calculation. Later modifica- 
tions were made to include other trace gas (Ciais et al., 1995; Conway et al., 1994). In this model, the atmospheric transport is based on a Climatology of monthly air movements. The second model is an off-line 3D model developed by Heimann and Keeling (1989) at the MaxPlanck-Institute fur Meteorologie of Hamburg, which has been extensively used to analyse the global $\mathrm{CO}_{2}$ budget (Heimann and Keeling, 1989; Heimann et al., 1989; Keeling et al., 1989; Ramonet, 1994). It is an off-line transport model based on observed meteorological parameters with a 12 hour time step. Neither model includes a dynamic and explicit formulation of the planet boundary layer (PBL).

We present here a protocol to compare these models, based on three experiments $(\mathrm{A}, \mathrm{B}, \mathrm{C})$ that make it possible to point out to the specific abilities of each model in simulating the mixing of atmospheric $\mathrm{CO}_{2}$.

Experiment $A$. We evaluate how the two models transport $\mathrm{CO}_{2}$ emitted by the same source of fossil fuel emissions. This provides a simple basis of comparison which is directly relevant to the $\mathrm{CO}_{2}$ problem and allows us to fully analyse transport patterns for the two models.

Experiment $B$. We use a $\mathrm{CO}_{2}$ scenario including terrestrial and oceanic fluxes. This set of "all but fossil-fuel" $\mathrm{CO}_{2}$ sources and sinks, which includes the full seasonal cycle, was inferred from the atmospheric NOAA/CMDL observations by Conway et al. (1994) with the $2 \mathrm{~d}$ model we use here, run in an inverse mode. This $2 \mathrm{D}$ inverse method gives a set of fluxes containing all the implied processes but does not allow to fully separate them. For example, terrestrial part of this set includes photosynthetic and respiration fluxes as well as the release by biomass burning, but we cannot study each component separately. We perform different simulations with the 3D model, using different longitudinal distributions of sources and sinks. Comparing these runs with each other allows us to evaluate and discuss the impact of the longitudinal distribution of the sources and sinks on the zonal profile of $\mathrm{CO}_{2}$. We also compare these runs with the results of the 2D model.
Experiment $C$. We deal with the full $\mathrm{CO}_{2}$ budget in the atmosphere using a complete $\mathrm{CO}_{2}$ scenario which includes fossil emissions as well as terrestrial and oceanic uptake $(C=A+B)$. Specifically, we perform a mass balance inversion of the $\mathrm{CO}_{2}$ fluxes in latitude and time with the $3 \mathrm{D}$ model using as an input the smoothed latitudinal profile of atmospheric measurements for the period 1990-1993.

After a brief description of the two models involved in this study (Section 2), the results of the fossil fuel simulation are analysed in terms of transport discrepancy between the two models (Section 3). Then, we deal with the other part of the atmospheric carbon cycle, an "all but fossilfuel" scenario (Section 4), before performing an inverse calculation of the complete latitudinal profile of $\mathrm{CO}_{2}$ (section 5). Finally, we discuss the implications of several modelling uncertainties for the balance of the carbon budget.

\section{Description of the two models}

\subsection{D model (PMTmodel)}

This model initially developed by Plumb and Mahlman (1987) will be hereafter referred to as the PMT model. The same transport fields have also been used by Enting and Mansbridge (1989). The atmosphere is represented by 10 vertical pressure levels and 20 latitudinal bands of equal area (Table 1), with a current time step of $6 \mathrm{~h}$. There are monthly fields of advective motion (stream function) and a spatially varying diffusion tensor, which describes the effects of eddy mixing via a flux-gradient parametrization. Together, they describe the average monthly and zonal mean transport of the GFDL general circulation model (Mahlman and Moxim, 1978), as determined by simulating two artificial tracers (Plumb and Mahlman, 1987). The lower vertical diffusivities between $1000 \mathrm{mb}$ and $800 \mathrm{mb}$ have been increased by a factor of 2 compared to the initial version of the PMT model as proposed by Tans et al. (1989), which has the effect to improve the simulated seasonal cycle of $\mathrm{CO} 2$ in the vertical dimension. In addition, a convective mixing was explicitly added later to the vertical transport at each time step (Brost and Heimann, 1991). 
Table 1. Main characteristics of the PMT and the TM2 model

\begin{tabular}{lll}
\hline \multicolumn{1}{c}{ Characteristics } & \multicolumn{1}{c}{ PMT model (2D) } & TM2 model (3D) \\
\hline horizontal resolution & 20 latitudinal sinus bands & $7.5^{\circ} \times 7.5^{\circ}$ \\
& (same area) & $(48 \times 25$ grid points) \\
vertical resolution & 10 equidistant sigma layers & 9 sigma layers \\
winds & climatology & ECMWF observed winds \\
advection & advective/diffusive scheme & slope scheme \\
& (Plumb and Mahlman, 1987) & (Russel and Lerner, 1981) \\
convection & adjustment & convective clouds from \\
& (Brost and Heimann, 1991) & (Tiedtke, 1989) \\
vertical diffusion & advective/diffusive scheme & stability dependent diffusion \\
& (Plumb and Mahlman, 1987) & (Louis, 1979) \\
winds timestep & monthly mean (interpolated) & $12 \mathrm{~h}$ \\
computing timestep & $6 \mathrm{~h}$ & $3 \mathrm{~h}$
\end{tabular}

\subsection{D model (TM2 model)}

Originally developed at NASA-GISS by Russel and Lerner (1981), this model has been further revised by Heimann et al. (1989-TM1, 1995-TM2). The version of the TM2 model used in this study has 9 vertical levels in sigma coordinates that extend from the surface to $10 \mathrm{mb}$. The horizontal resolution is of $7.5^{\circ}$ by $7.5^{\circ}$ (Table 1 ) with a time step of $3 \mathrm{~h}$. It is an off-line transport model based on meteorological fields analysed by the European Center for Medium Weather Forecast (ECMWF) every $12 \mathrm{~h}$. Both shallow and deep convective mixing together with vertical diffusion are calculated at each time step (Louis, 1979; Tiedtke, 1989).

\subsection{Main differences}

There are important specific differences between the two models. First, in a 2D model, the zonal mixing of air masses is assumed to be fast compared to meridional mixing. This is the main justification for two-dimensional modelling of atmospheric transport. Such an assumption is not necessary in the TM2 model where the longitudinal air flow is explicitly accounted for. Secondly, the PMT model has climatological wind fields whereas TM2 has analysed ones, based on meteorological observations. As a consequence, the PMT model has no inter-annual differences in the atmospheric transport. Thirdly, both models differ in their advection schemes. The PMT model diffusive transport coefficients are 4-element ten- sors, of which the anti symmetric part contributes to the effective advective transport, and the symmetric part represents purely diffusive mixing (Plumb and Mahlman, 1987). The TM2 model uses only the horizontal advection fields from the ECMWF meteorological analysis, with the vertical advection being calculated from mass conservation in each grid box. Last, the 2D-model has a simple parametrisation of convective mixing (Brost and Heimann, 1991) whereas the TM2 model includes a more realistic deep and shallow convection based on clouds mass flux scheme (Tiedtke, 1989), and vertical diffusion (Louis, 1979)

\subsection{Validation of the two models with inert tracers}

The vertical transport of the PMT model has been tested with the attenuation of the seasonal cycle of $\mathrm{CO}_{2}$ and the TM2 model has been tested using the ${ }^{222} \mathrm{Rn}$ measurements compiled by Liu et al. (1984). The vertical diffusivities of the PMT model were also compared to those derived by Liu et al. (1984). In the PMT model, Tans et al. (1989) observed a better agreement with the observations when the vertical diffusivities in the lowest two model layers were doubled. In the TM2 model, Ramonet (1994) found that the vertical attenuation of ${ }^{222} \mathrm{Rn}$ concentrations is rather well represented in summer but that the model overestimates the observations above $6 \mathrm{~km}$ in winter. Using detailed airborne measurements made in the campaign TROPOZ II, Ramonet et al. (1996) 
have also shown that TM2 reproduced most of the observed synoptic variations in ${ }^{222} \mathrm{Rn}$, although it overestimates the concentrations in the mid-to-high troposphere. This discrepancy indicates a too vigorous vertical mixing in the TM2 model. This tendency is confirmed by a recent inter-comparison of several cumulus parametrizations in chemical transport models (Mahowald et al., 1995).

The large-scale transport in the two models has been tested against ${ }^{85} \mathrm{Kr}$ measurements (Heimann and Keeling, 1989; Tans et al., 1989). Generally, good agreement with observations was found. For the PMT model, the inter-hemispheric exchange time (i.e. the difference of the ${ }^{85} \mathrm{Kr}$ inventory in both hemispheres divided by the flux across the equator) is very close to the value of 1.1 year obtained by Jacob et al. (1987) with the GISS transport model (Prather et al., 1987). For the TM2 model, no ${ }^{85} \mathrm{Kr}$ calculation was published, but the figure is close to TM1 (Ramonet, unpublished results) with a value of 1.3 years, suggesting that the mixing between both hemispheres is slightly faster in the real world than as represented by the model (Heimann and Keeling, 1989). Note that a tracer like ${ }^{85} \mathrm{Kr}$ is not analogous to $\mathrm{CO}_{2}$ because it is emitted only at a few specific locations with no seasonality, although it gives a good idea of inter-hemispheric mixing in atmospheric models. However, ${ }^{85} \mathrm{Kr}$ may be a close match to the fossil-fuel $\mathrm{CO}_{2}$ tracer for which we calculate the inter-hemispheric exchange times in Section 3.

\section{Experiment A, fossil fuel simulation}

We run both models with the same fossil fuel source of $\mathrm{CO}_{2}$ prescribed by Tans et al. (1989). In the TM2 model, we distribute this zonal source in longitude according to the distribution of the fossil fuel emissions calculated by Marland et al. (1985). The total magnitude of this source is $508 \mathrm{Tmol}$ $\mathrm{yr}^{-1}$ (6.09 GtC $\mathrm{yr}^{-1}-1 \mathrm{Tmol}=10^{12} \mathrm{~mol}=0.012$ $\mathrm{GtC}$ ). We have run both models during four years, following the protocol of Transcom I (Rayner and Law, 1995). The first three years are a spin-up and the results are presented for the fourth year of simulation. For TM2, we repeat identically the 1990 meteorological winds and convection during the four years of simulation.

Fig. 1 presents the zonal annual surface concen-

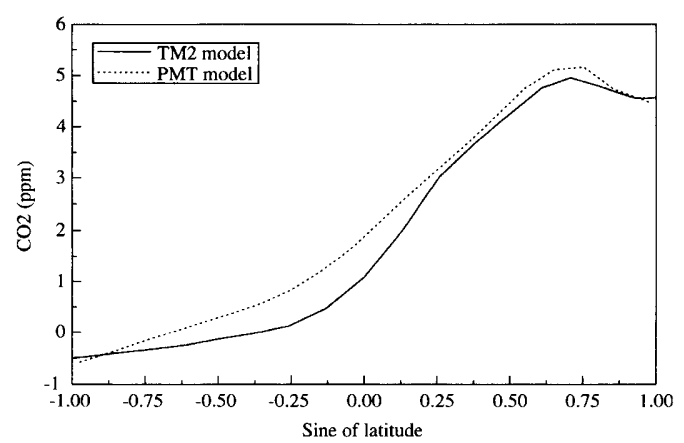

Fig. 1. Zonal annual mean surface concentration in fossil $\mathrm{CO}_{2}$ (experiment A) for the fourth year of simulation. The PMT model (dashed line) is compared to the TM2 model (solid line). Curves are relative to the global mean mixing ratio for January which explains negative concentrations.

tration in fossil $\mathrm{CO}_{2}$ as calculated in both models. The concentrations are calculated relative to the global mean atmospheric mixing ratio found by the models in January of the 4th year of simulation. This reference is identical in both models. Mixing ratios in the PMT model and the TM2 model are interpolated at the same $950 \mathrm{mb}$ pressure level. North of $30^{\circ} \mathrm{N}$, the two models transport fossil $\mathrm{CO}_{2}$ in a relative similar manner at the surface. Across the equator, the TM2 model produces a steeper decline in fossil $\mathrm{CO}_{2}$ than the PMT model, indicating that at the surface level, the north/south mixing in the inter-tropical zone is weaker in TM2. In the southern extra-tropics and at high southern latitudes, the opposite behaviour is observed since the TM2 model appears to mix fossil $\mathrm{CO}_{2}$ more vigorously than the PMT model.

To fully compare the large-scale meridional transport in the two models, we must consider all the vertical layers. It is common practice to use vertical column averages of the north to south fluxes to estimate the strength of the transport. However, in this steady state experiment, where sources are in equilibrium, the model fluxes across any vertical zonal column are identical in both models. The resulting latitudinal profile is the profile that is necessary to produce such a flux for both models. Thus, we have calculated in Table 2 the interhemispheric exchange time defined as the north minus south difference in mean concentration divided by the flux across the equator. Table 2 
Table 2. Inter-hemispheric exchange time for both models (years), defined as the difference between the concentration means in the two hemispheres (global) or the concentration means at surface in the two hemispheres (surface), divided by the concentration flux across the equator

\begin{tabular}{lcc}
\hline Model & Global & Surface \\
\hline TM2 model & 1.24 & 1.56 \\
PMT model & 0.97 & 1.36 \\
\hline
\end{tabular}

shows the exchange times both for the global atmosphere and also for the surface level only. Table 2 indicates that the PMT model has a faster north/south mixing than TM2 not only at the surface level $(\sim+15 \%)$ but also averaged through the vertical $(\sim+25 \%)$. Fig. 2 a compares the modelled concentration fields in both hemispheres. The PMT model exhibits a smaller global north to south difference than the TM2 model $(\sim 20 \%$ and $40 \%$ smaller respectively at $500 \mathrm{mb}$ and 200 $\mathrm{mb}$ ). This difference is only $15 \%$ at the surface (Fig. 1) and is fully consistent with the differences in ${ }^{85} \mathrm{Kr}$ exchange times (section 2). Fig. $2 b$, c display the zonal annual concentration in fossil $\mathrm{CO}_{2}$ for both models. At mid-northern latitudes, above the main source region, $\mathrm{CO}_{2}$ emitted at the surface penetrates higher in altitude in TM2 than in PMT, resulting in a smaller gradient across the tropopause for TM2. This means a stronger intrahemispheric mixing for TM2 than for the PMT model, above main source region. We miss here a specific tracer of the intra-hemispheric exchange to quantify more precisely this effect. At the equator, the southward flow of $\mathrm{CO}_{2}$ is detected for TM2 mostly in the mid-to-high troposphere ( $\sigma 0.2$ to 0.6 ), whereas the PMT model instead exhibits a tongue of northern hemisphere air flowing to the south at sigma $\sim 0.2$. Note also that, across the equator, the isolines are closer to one another for TM2 than for the PMT model. This means that TM2 also exhibits the "braking" effect that was seen at the surface (Fig. 1) along the whole vertical. These differences in transport patterns are consistent with the remarks made previously about horizontal and vertical validation of the two models (Heimann and Keeling, 1989; Tans et al., 1989).

Apart from the annual average transport pat-
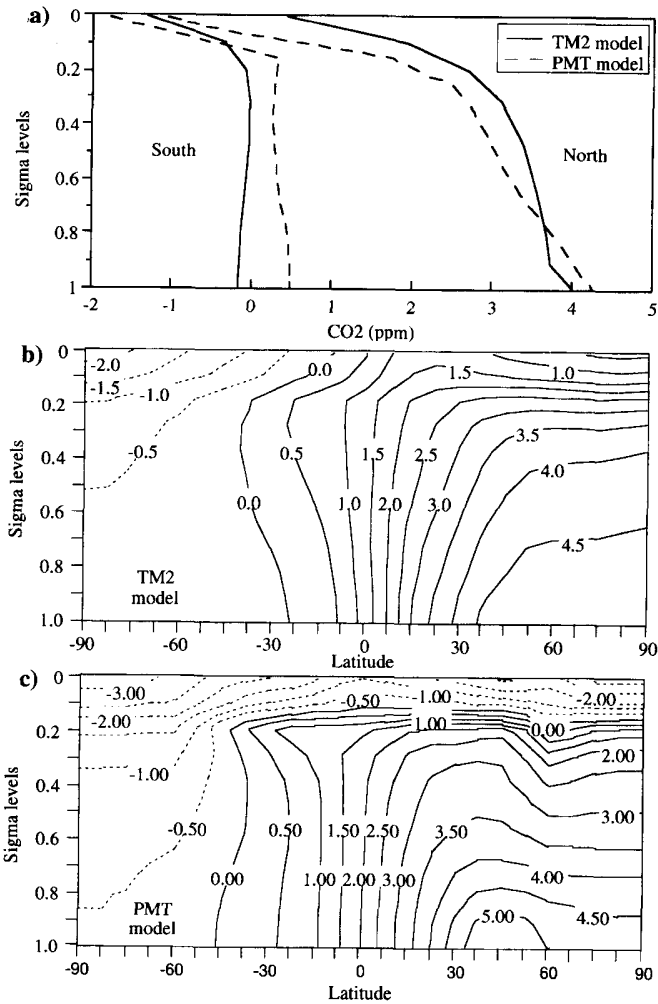

Fig. 2. Vertically intagrated annual mean concentration in fossil $\mathrm{CO}_{2}$ for the fourth year of simulation (in ppm). (a) Annual northern and southern hemisphere mean concentration for the PMT model (dashed line) and for TM2 (solid line). The zonal annual mean concentration for the TM2 model (b) is also compared to the one for the PMT model (c). All the curves are relative to the global mean mixing ratio for January, which explains negative concentrations.

terns, we examined the variations in the north/south $\mathrm{CO}_{2}$ profile due to inter-annual changes in the ECMWF fields used in TM2. Fig. 3 plots the annual zonal mean concentrations in fossil $\mathrm{CO}_{2}$ at the surface obtained with the same fossil fuel source but with specific ECMWF fields from 1990 to 1994 . We find that the maximum difference over the five-year period does not exceed $0.2 \mathrm{ppm}$ and is maximum over mid-north latitude source region. This difference maybe either due to climate variations or to ECMWF analysis of meteorological fields (ECMWF analysis model has changed in 1993). We have also performed a sensitivity test to check the respective influence of 


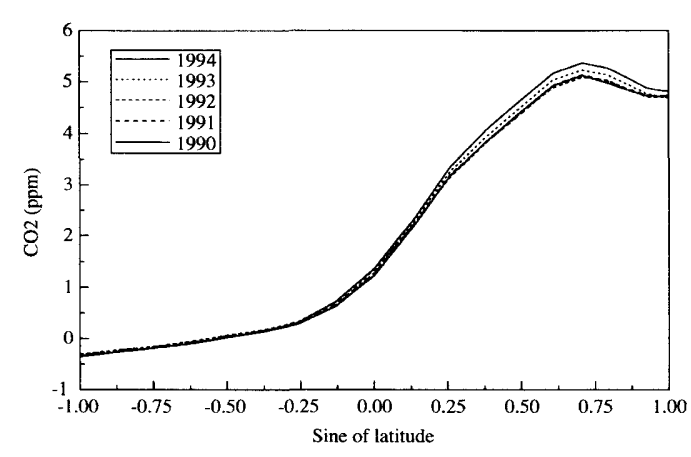

Fig. 3. Influence of different years of the ECMWF meteorological fields on the modelled zonal annual surface mean concentration in fossil $\mathrm{CO}_{2}$ (experiment $\mathrm{A}$ ). The model used is the TM2 model. Input fluxes are fossil fuel emissions from Tans et al. (1989), distributed in longitude according to Marland et al. (1985). All curves are relative to the global mean mixing ratio for January of the corresponding year.

winds and convection on these variations. Roughly, we keep either convection fields or wind fields constant during the simulation to evaluate the respective part of each field in the differences of latitudinal profiles. Averaged over the 5-year period, it appears that this influence for both winds and convection is of the same order.

Consequently, the influence of inter-annual variability of meteorological ECMWF fields on the annual mean $\mathrm{CO}_{2}$ latitudinal profile is weak compared to the differences between the two models.

\section{Experiment B, "All but fossil-fuel" simulation}

The goal of this section is to evaluate the influence of the longitudinal distribution of $\mathrm{CO}_{2}$ sources and sinks in the 3D model, using an "all but fossil-fuel" scenario which includes both terrestrial and oceanic fluxes with their respective seasonal cycles.

We have used the $\mathrm{CO}_{2}$ sources and sinks inferred from the atmospheric NOAA-CMDL observations, using the PMT model run in an inverse mode. A detailed presentation of the NOAA-CMDL $\mathrm{CO}_{2}$ measurements is given in Conway et al. 1994. The inverse calculation of the fluxes from flasks measurements was developed by Tans et al. (1989), and has been further used by Conway et al. (1994) and by Ciais et al. (1995). Briefly, the individual time series of flask data at each site of measurement is first smoothed in the time domain using the curve fitting and data extrapolation method detailed in Masarie and Tans (1995). Gaps in the data are filled by using stable characteristics of nearby sites. A weekly latitudinal fit of the smoothed time series at each site is then constructed, in which a greater weight is given to sites with a higher number of sample pairs. We subtract the well known fossil fuel emissions from the inferred surface fluxes to obtain a 2D-set of "all fluxes excepting fossil fuel" (terrestrial biosphere +ocean). This zonal source is plotted on Fig. 4a: the equatorial release and the midnorthern latitude sink are clearly identified, the others latitudinal regions being small sources or sinks.

We apply these zonal fluxes to the TM2 transport for four consecutive years, using a three year spin up, and archiving the simulated $\mathrm{CO}_{2}$ concentrations in the subsequent four years. These years correspond to the period $1990-93$ period for $1 /$ the annual meteorological fields and $2 /$ the $\mathrm{CO}_{2}$ zonal fluxes deduced from the NOAA/CMDL observations. Running zonal $\mathrm{CO}_{2}$ fluxes in a $3 \mathrm{D}$ model raises the problem of the longitudinal distribution. Because the meridional transport across the Equator is not uniform in longitude (Barry and Chorley, 1987), the simulated $\mathrm{CO}_{2}$ latitudinal profiles in TM2 should depend strongly upon the sources and sinks positions in longitude. In order to examine such interactions between fluxes and southward transport, we have performed 3 simulations. In run B1, we distribute the zonal fluxes inferred from the $2 \mathrm{D}$ inversion evenly in longitude (Fig. 4a). In run B2 we use two uniform distributions in longitude, one for land and one for the oceans (Fig. 4b). The separation of the terrestrial

Fig. 4. Annual mean flux scenarios for the $3 \mathrm{~d}$ simulations (year 1992) : in run B1 (a) we distribute the zonal fluxes inferred from the 2D inversion evenly in longitude. In run B2 (b) we also use a uniform distribution in longitude but make a distinction between ocean and land fluxes based on independent information brought by $\delta^{13} \mathrm{C}$ measurements. In run B3 (c), we distribute terrestrial fluxes proportionaly to the annual mean NPP from Ruimy et al. (1994), keeping zonal oceanic fluxes. 

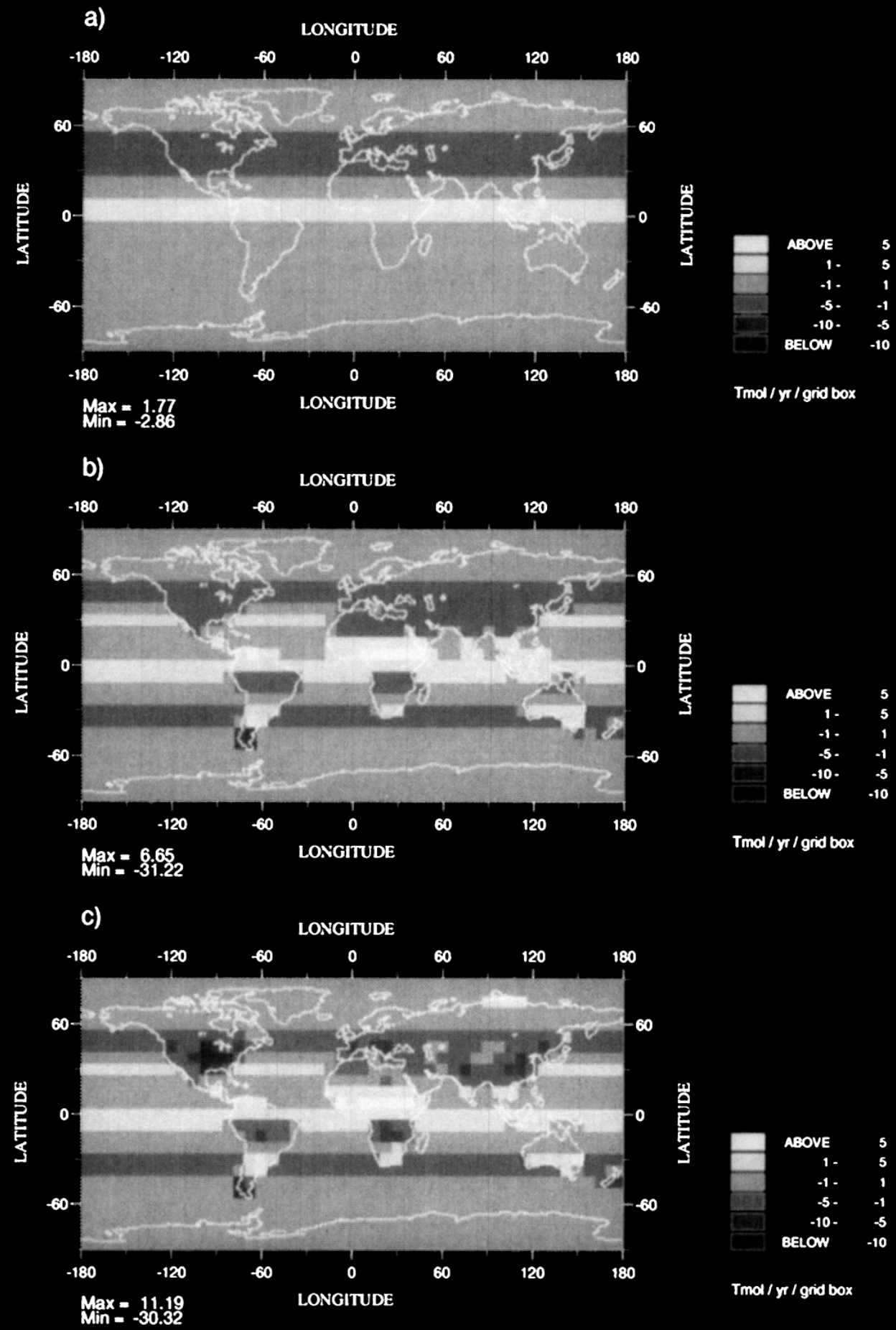

$\operatorname{Tmol} / \mathrm{y} /$ grid box 
fluxes is done according to a double deconvolution of $\delta^{13} \mathrm{C}$ measurements by Ciais et al. (1995b). In run $\mathrm{B} 3$, we distribute terrestrial fluxes proportionally to the annual mean NPP from Ruimy et al. (1994), keeping the oceanic fluxes zonal (Fig. 4c). This represents a step towards a more realistic geographical distribution of the $\mathrm{CO}_{2}$ fluxes.

Fig. 5a plots the zonal mean surface $\mathrm{CO}_{2}$ profile obtained in the PMT model and in the three TM2 runs. All runs B1, B2 and B3 exhibit zonal concentration fields relatively close to each other but which differ significantly from the PMT model run. The largest difference between TM2 and PMT occurs at mid-northern latitudes $(0.7 \mathrm{ppm}$ between PMT and run B1 and 0.5 ppm between PMT and runs B2/B3). Another significant discrepancy can be observed in the tropics where the $3 \mathrm{D}$ runs are very close to each other but differ from the $2 \mathrm{D}$ run by $0.5 \mathrm{ppm}$ around the equator. This behaviour corresponds to a weaker inter-tropical hori-
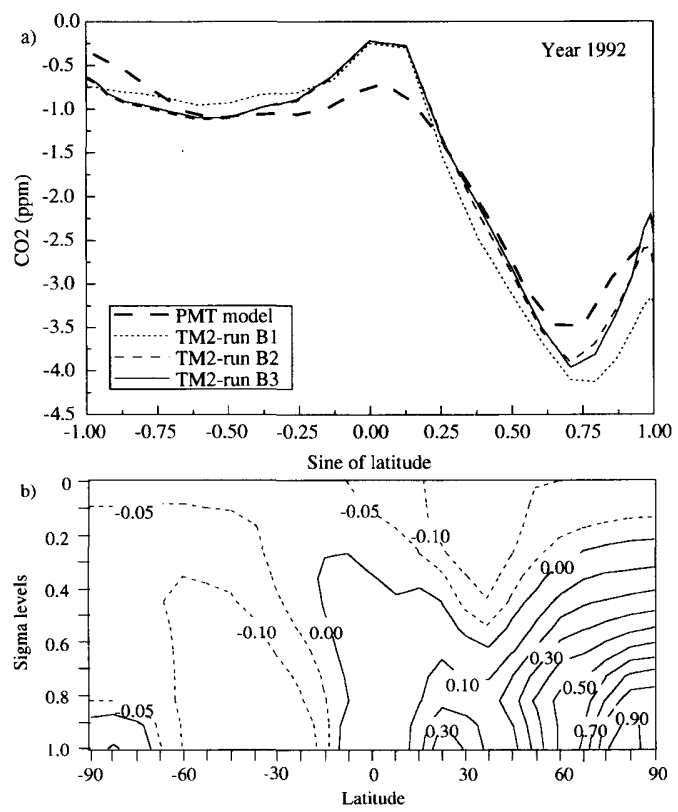

Fig. 5. Zonal annual mean concentration of $\mathrm{CO}_{2}$ for the different "all but fossil-fuel" simulations (year 1992). a) Latitudinal surface concentrations in ppm. We compare the PMT model (bold dashed line) with TM2-run B1 (dashed line), TM2-run B2 (dotted line) and TM2-run B3 (solid line). b) Vertically integrated differences between TM2-runB3 and TM2-run B1 (ppm). All the curves are relative to the global mean mixing ratio for January 1992. zontal mixing in TM2, and a higher northern midlatitude convection compared to the PMT model, as underscored in section 3. Consequently, matching the observed $\mathrm{CO}_{2}$ latitudinal profile with the TM2 transport would require a smaller sink at mid-northern latitudes and a smaller source in the tropics than those deduced by Conway et al. (1994) and Ciais et al. (1995b) with the PMT model. We will quantify those concentrations differences in term of $\mathrm{CO}_{2}$ fluxes in Section 5.

Furthermore, the impact of longitudinal distribution of the sources and sinks is not negligible. Run B2 and B3 are close to each other but they differ significantly from run B1 in extra-tropical regions. This suggests that the ocean versus continent partitioning has a greater effect on the interhemispheric $\mathrm{CO}_{2}$ latitudinal profile than the partitioning as a function of NPP within the continents as it implies greatest differences in the initial fluxes (see Fig. 5). So, we restrict our discussion now to runs B1 and B3. Some possible mechanisms can be found to explain the differences between runs B1 and B3. At mid-northern latitudes, biospheric uptake has a strong seasonality, with a maximum in summer when vertical mixing over the continents is strongest. Moreover, convection is much greater above land areas than above the oceans at these latitudes. Therefore, distributing the midnorthern sink mainly on the continents (run B3) induces a stronger vertical dilution of this sink and consequently reduces the depletion of surface concentrations compared to zonal run B1. To illustrate this point, Fig. $5 \mathrm{~b}$ show the vertical section of the zonal annual mean difference between run B3 and run B1. The surface and the low troposphere in mid and high northern latitudes are less depleted in "all but fossil fuel" $\mathrm{CO}_{2}$ in run $\mathrm{B} 3$ than in run $\mathrm{B} 1$ (strong positive anomaly) due to the vertical dilution by convection. Consequently, a small negative anomaly is found in the rest of the atmosphere. In the tropics, a second effect occurs which could compensate the first one. In fact, the strong inter-tropical source (see Fig. 4) is located more over equatorial Africa in run B3, instead of being distributed on both oceans and continents for the run B1. Now, if we look closely at the position of the ITCZ in the tropics (Barry and Chorley, 1987), we find that for a large part of the year, especially in boreal summer, the equatorial Africa is at the north of the equator trough. In contrast, the ITCZ is more 
stable over oceans, and corresponds to a preferential injection of equatorial fluxes into the southern hemisphere. Thus, more tracer is kept in the northern tropics in run B3 than in run B1; this mass is vertically diluted by strong equatorial Africa convection and transport northward by Hadley cell. Finally, by distributing the equatorial source in longitude we reduce the efficiency of inter-hemispheric exchange in TM2. This reduction can be observed in Fig. 5a where one may notice a flip-flop effect at the surface between the two hemispheres : values for run B3 are higher than those for run B1 in the northern hemisphere but lower in the southern hemisphere. This effect is weak but qualitatively significant.

\section{Experiment $\mathrm{C}$, full $\mathrm{CO}_{2}$ cycle experiment}

The goal of this section is to evaluate the influence of modelled transport on the carbon budget using a complete $\mathrm{CO}_{2}$ scenario which includes fossil emissions as well as terrestrial and oceanic fluxes.

We use the same ocean and land $\mathrm{CO}_{2}$ sources and sinks as in experiment $\mathrm{B} 3$, adding fossil fuel emissions prescribed by Tans et al. (1989), distributed in longitude over the continents (experiment A) and actualised for the years 1990-1993 (Conway et al., 1994). We run this complete $\mathrm{CO}_{2}$ scenario with TM2 (run $\mathrm{C}=\operatorname{run} \mathrm{B} 3+\operatorname{run} \mathrm{A}$ ). We also run the corresponding zonal scenario, inferred from the PMT inverse model, in the forward version of the same PMT model to verify that we obtain the observed latitudinal profile from which we originally started.

Fig. 6 plots the zonal annual surface concentration of $\mathrm{CO}_{2}$ obtained in the PMT model (observed meridional profile) and in the TM2 model (run C), for the year 1992. Fig. 6 thus combines the differences outlined in Fig. 1 and Fig. 5. In comparing Fig. 6 to Fig. 5, we find differences between the $2 \mathrm{~d}$ and $3 \mathrm{~d}$ model in the northern hemisphere more or less as expected from the discussion above. However, the discrepancy between the TM2 and PMT model in the tropics is not so apparent in Fig. 6. The fact that TM2 keeps more tracer emitted locally in the tropics (see Fig. 5) is partially compensated by a weaker advection of fossil $\mathrm{CO}_{2}$ coming from northern hemisphere at the surface (see Fig. 1). It is important to translate

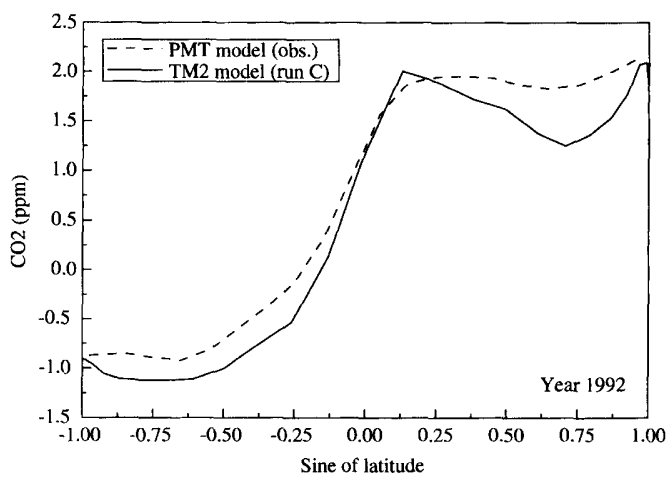

Fig. 6. Zonal annual surface mean concentration of $\mathrm{CO}_{2}$ for the PMT model and the TM2 model (year 1992): we compare observations (dashed line) to the TM2-run C (solid line). Both curves are referred to the global mean mixing ratio for January 1992.

such differences in the modelled $\mathrm{CO}_{2}$ concentrations between the TM2 and the PMT model in terms of net surface fluxes. In other words we must estimate what zonal $\mathrm{CO}_{2}$ fluxes are needed in the TM2 model to match the observed zonally averaged latitudinal profile. We describe below an iterative mass balance inversion of the smoothed observed $\mathrm{CO}_{2}$ latitudinal profile using the TM2 winds. The question is to determine what zonal sources in the $3 \mathrm{D}$ model give the same $\mathrm{CO}_{2}$ zonal mean as the original $2 \mathrm{D}$ profile.

The inverse calculation is constrained by weekly zonal meridional profiles in the $\mathrm{CO}_{2}$ mixing ratio $C_{2 \mathrm{~d}}(\lambda, t)$, where $\lambda$ represents the different latitude bands and $t$ the weekly interval. For every TM2 model time step $(3 \mathrm{~h})$, we calculate the instantaneous zonal profile $C_{3 d}(\lambda, t)$ in the 3D model, from which we subtract $C_{2 \mathrm{~d}}(\lambda, t)$ to obtain a flux correction factor $\beta$ defined as:

$$
\left.\beta=\left(C_{3 \mathrm{~d}}\right)(\lambda, t)-C_{2 \mathrm{~d}}(\lambda, t)\right) \times f \times \Gamma_{\mathrm{m}}
$$

where $f$ is the conversion factor between ppm and GtC $\left(2.122\right.$ GtC.ppm $\left.{ }^{-1}\right)$ and $\Gamma_{\mathrm{m}}$ is the air mass contained in the latitude band $\lambda$ divided by the total mass of the atmosphere. The value of $\beta$ in each latitude band $\lambda$ is distributed evenly in longitude over the TM2 model grid in order to correct the source field and to yield the required atmospheric concentrations $C_{2 \mathrm{~d}}(\lambda, t)$. This flux correction starts in the first year of spin-up to ensure a steady state regime after the fourth year. 
Numerically, our flux correction proved to be unstable when the initial value of $\beta$ was too large, roughly of the order of the source to be corrected. To avoid this problem, $\beta$ is attenuated during spin-up, by $50 \%$ on the 1 st year of correction and by $25 \%$ on the 2 nd year. The corrected fluxes are saved at each time step for other possible iterations. In order to minimise the difference between the inverted concentrations and the observed "target" concentration fields, we iterate this process several times with the sources as in run $\mathrm{C}$.

Using this methodology, we obtain a zonal carbon budget with TM2 which is compared, for the year 1992, to the sources inferred by Conway et al. (1994) with the PMT model (Fig. 7). Table 3 provides the resulting fluxes averaged over wide latitude bands for 1990-1993. In the northern hemisphere, Fig. 7 shows that the position in latitude of the sources and sinks is approximately the same in the two models. However, its strength is different. North of $65^{\circ} \mathrm{N}$, the initial source is

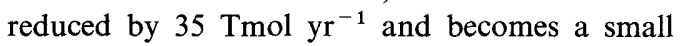

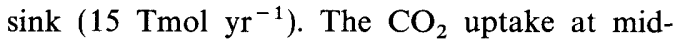
northern latitude is $80 \mathrm{Tmol} \mathrm{yr}^{-1}$ smaller as given by TM2 than by the PMT model for period 1990-93. This difference almost reaches $100 \mathrm{Tmol}$ $\mathrm{yr}^{-1}$ in 1992. The equatorial source is also reduced by $40 \mathrm{Tmol} \mathrm{yr}^{-1}$ over the period $1990-93$ when inferred by TM2. In addition, TM2 shifts the equatorial source southward by roughly $10^{\circ}$ (from $10^{\circ} \mathrm{N}$ to $0^{\circ}$ ) compared to PMT. Since TM2 has a weaker surface transport across the equator (Fig. 1), the inverse calculation must inject $\mathrm{CO}_{2}$ further to the south to match the observations in this latitude band. The $\mathrm{CO} 2$ uptake at mid northern latitudes and the release in the northern tropics as inferred by mass balance calculations are correlated because their relative strength depends on the intensity of the mixing within the Northern Hemisphere. Thus, Fig. 7 suggests, as Fig. 2, that the PMT model has a weaker intra-hemispheric mixing than TM2, although it has a stronger transport across the equator. In the southern extra-tropics, the fluxes derived from both models do not differ by more than $15 \mathrm{Tmol}^{-1}$. Finally, note that running experiment $\mathrm{C}$ with $(\mathrm{B} 1+\mathrm{A})$ instead of $(B 3+A)$ leads to differences in the inferred carbon budget lower than $15 \mathrm{Tmol} \mathrm{yr}^{-1}$. The differences observed in experiment $\mathrm{B}$ on the latitudinal profiles and due to the longitudinal distribution of fluxes are smoothed by the zonal inversion performed here.

Table 3. Zonal carbon budget inferred from the TM2 model for the period 1990-1993

\begin{tabular}{lcrrrrrr}
\hline Year 1990 & $90 \mathrm{~S}-20 \mathrm{~S}$ & $20 \mathrm{~S}-0$ & $0-20 \mathrm{~N}$ & $20 \mathrm{~N}-65 \mathrm{~N}$ & $65 \mathrm{~N}-90 \mathrm{~N}$ & GLB & ACC \\
\hline PMT model & -120 & $\mathbf{4 0}$ & $\mathbf{1 2 0}$ & $-\mathbf{3 6 0}$ & 35 & -285 & 225 \\
TM2 (run C) & -130 & $\mathbf{9 0}$ & $\mathbf{3 5}$ & $-\mathbf{2 7 0}$ & -10 & -285 & 225 \\
Year 1991 & & & & & & & \\
PMT model & -125 & $-\mathbf{2 5}$ & $\mathbf{1 6 0}$ & $\mathbf{- 3 6 5}$ & 40 & -315 & 200 \\
TM2 (run C) & -135 & $\mathbf{4 0}$ & $\mathbf{6 0}$ & $-\mathbf{2 8 0}$ & $<5$ & -315 & 200 \\
Year 1992 & & & & & & & \\
PMT model & -125 & -25 & $\mathbf{1 6 0}$ & $\mathbf{- 4 1 0}$ & 15 & -385 & 125 \\
TM2 (run C) & -120 & 10 & $\mathbf{6 0}$ & $\mathbf{- 3 1 5}$ & -20 & -385 & 125 \\
Year 1993 & & & & & & & \\
PMT model & -110 & $<5$ & $\mathbf{1 2 0}$ & $\mathbf{- 3 5 0}$ & $<5$ & -340 & 175 \\
TM2 (run C) & -115 & 45 & $\mathbf{5 0}$ & $\mathbf{- 3 1 0}$ & -20 & -340 & $\mathbf{1 7 5}$ \\
\hline
\end{tabular}

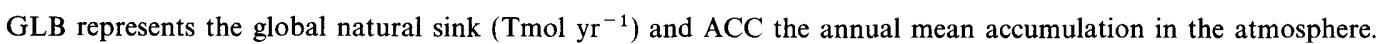
Fossil fuel emissions are, respectively, of 508, 516, 508 and $513 \mathrm{Tmol} \mathrm{yr}^{-1}\left(6.09,6.19,6.09\right.$ and $6.15 \mathrm{GtC}_{\mathrm{yr}}^{-1}$, respectively) for the years 1990, 1991, 1992 and 1993. Main differences are in bold. We do not write figures smaller

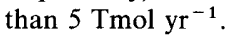




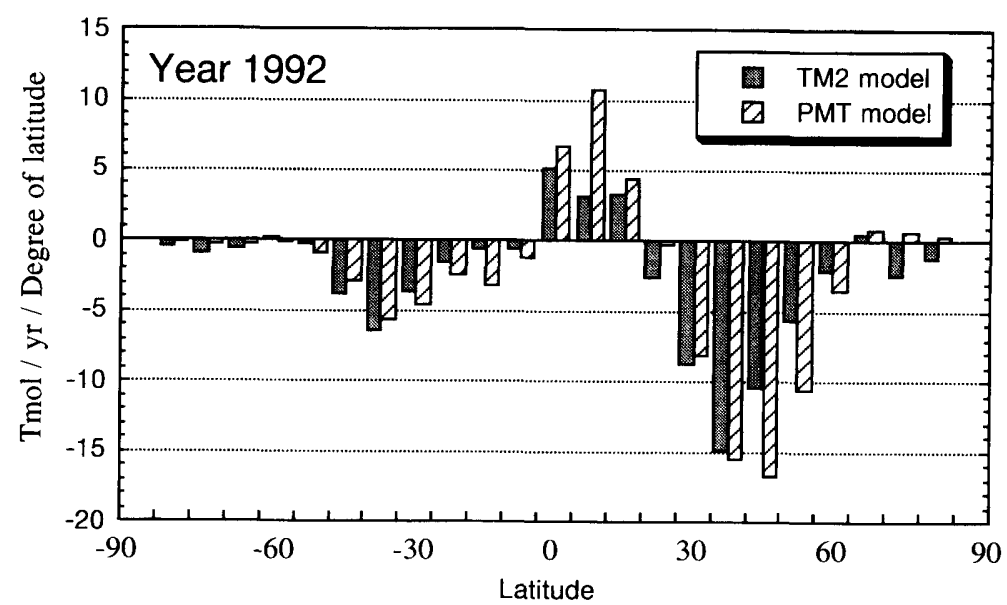

Fig. 7. Corrected $\mathrm{CO}_{2}$ surface fluxes to match observed zonal concentration with the TM2 model (Tmol yr ${ }^{-1}$ degree $^{-1}$ ). Annual means for year 1992. Fossil fuel emissions have been subtracted. Dashed bars represent the initial $\mathrm{CO}_{2}$ fluxes found by Conway et al. (1994). Plain bars are the result of a zonal mass balance inversion performed in this study with the TM2 model.

\section{Discussion}

This work shows that there are important changes in the global carbon budget required when moving from one atmospheric transport model (PMT) to another (TM2). This result is very important as it implies that the uncertainty due to modelled transport is a major uncertainty for the inversion studies as well as the scarcity of the measurement network. Future 3d inversions should take into account this result. Particularly, inter calibrations between transport models must be pursued to establish more rigorously the mixing behaviour of different models in the vertical dimension. Even more importantly, more data are needed in the vertical to constrain the models better. Dealing almost exclusively with observed surface mixing ratios makes the inferred carbon budget too model-dependent. As an example, if we only refer to the surface layer, we have compared two relatively similar models when looking at the wide range of variations between Transcom models (Rayner and Law, 1995). But, by analysing their behaviour on the vertical, we showed that they lead to different structures of $\mathrm{CO}_{2}$ concentrations and thus to different carbon budget.

Recently, another cause of uncertainty in the modelled transport has been raised by Denning et al. (1995). They show that the simulated north/south $\mathrm{CO}_{2}$ latitudinal profile due to sea- sonal exchange with terrestrial biota ( 2 ppm from pole to pole) is nearly half as strong as that imposed by the fossil fuel emissions $(4.5 \mathrm{ppm})$ when using a GCM (CSU GCM) including a parametrisation of the Planet Boundary Layer (PBL). Neither of the two models in this study has an explicit and complete implementation of the PBL. However, one can say that the TM2 model has a crude boundary layer representation because the vertical diffusion coefficients calculated with the scheme of Louis et al. (1979) are dependent on the stability of air. The effect of this is limited both by the large time interval of ECMWF meteorological fields ( 12 hours) and by the relatively coarse vertical resolution of this version of the TM model close to the ground (roughly two levels between 0 and $1.2 \mathrm{~km}$ ). We expect that the addition of a PBL in TM2 (or even just a few more levels close to the surface) would increase significantly the mid-northern latitude sink of $\mathrm{CO}_{2}$ inferred by the inversion because of the co-variation of vertical transport and the biological $\mathrm{CO}_{2}$ source/sink. When the vertical mixing is strong during summer $\mathrm{CO}_{2}$ sinks prevail, whereas during winter the $\mathrm{CO}_{2}$ sources dominate while the mixing is weak, allowing substantial build-up in the PBL. Rayner and Law (1995) show a tendency for the modelled latitudinal profiles to fall into two groups, with or without 
an explicit PBL, when dealing with seasonal exchange between atmosphere and land biota.

Finally, dealing with these two model uncertainties and different sets of recent $\delta^{13} \mathrm{C}$ measurements, it seems that the idea of a strong biospheric sink of $\mathrm{CO}_{2}$ in the northern hemisphere for the 1990s is not so controversial at the present time (GAIM conference, Garmish Partenkirshen, Germany, September 1995). The problem is to simulate properly the exchanges between atmosphere and land ecosystems for this period that seems very different from the 1980's for the carbon budget. We show that the uncertainty due to modelled atmospheric transport needs to be considered as well as other uncertainties, on sources and sinks or on measurements. In order to fully understand the atmospheric carbon cycle during the 1990's, we must reduce the uncertainty due to modelled transport and continue to work on the Biogeochemical mechanisms explaining ocean and terrestrial biosphere $\mathrm{CO}_{2}$ global uptake. This protocol is a step in this direction that allows to understand and quantify the transport differences existing between two models. An improvement would be to infer partitioned fluxes between ocean and land with TM2. To do so, the information given by $\delta^{13} \mathrm{C}$ measurements (Ciais et al., 1995) should be integrated in the TM 2 model.

\section{Conclusions}

In this paper, we evaluate one uncertainty introduced by modelled atmospheric transport on the balance of the carbon budget. To do so, we propose a protocol to compare the ability of two atmospheric transport models to account for the different components of the atmospheric carbon cycle. Using only fossil fuel emissions (experiment A) lead us to conclude that the PMT model has a stronger north/south mixing than TM2 by about $25 \%$ for the global atmosphere. Vertical mixing above source regions is found to be much weaker in the PMT model than in TM2. Analysing "all but fossil experiment" (experiment B) provide us with two important results. First, using the 3D transport of TM2 to carry sources and sinks inferred from the inverse PMT model leads to major differences in surface concentrations in the tropics and at mid-northern latitudes. Secondly, differences between the TM2 simulations we performed show that the influence of the distribution of sources and sinks in longitude has a significant effect on surface concentrations on both sides of the equator. This result implies that the zonal assumption made in $2 \mathrm{D}$ studies is not so robust even if the effect on surface concentrations is not as important as the previous effect of modelled transport. Finally, owing to a set of full $\mathrm{CO}_{2}$ fluxes (experiment $\mathrm{C}$ ), we find that using a $3 \mathrm{D}$ model with a weaker large-scale transport, we reduce the equatorial release by about $40 \mathrm{Tmol} \mathrm{yr}^{-1}$ and the mid the northern latitude sink by about $80 \mathrm{Tmol}$ $\mathrm{yr}^{-1}$ for the years $1990-1993$ when comparing to the initial 2D budget. These differences between $2 \mathrm{D}$ and $3 \mathrm{D}$ transport do not strictly speaking constitute an error, but rather a range of variation between two given models. We note however that this range is comparable in magnitude to the global data-based error range derived by Ciais

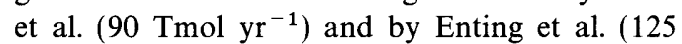

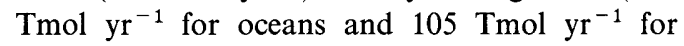
biota) on the carbon budget as inferred by ${ }^{13} \mathrm{C}$ isotopes.

This three-step protocol allows to derive the main differences on large-scale transport for two models and to quantify the discrepancy between atmospheric latitudinal profiles in terms of net $\mathrm{CO}_{2}$ surface fluxes.

\section{Acknowledgements}

This work is part of a close collaboration between the CFR and the LMCE. We acknowledge computing support from IDRIS and from CEA/LMCE. M.Heimann from Max-PlanckInstitute fur Meteorologie (Hamburg) is to be thanked for providing CFR with the TM2 model and for his interesting remarks about this work. At last, we would like to thank David Wu for his pertinent re-reading of this paper. This work is supported by the Programme National d'Etude de la Dynamique du Climat (PNEDC), and the Comission of European Community program No. EV5V CT92-0120. This is contribution no. 1784A of the CFR and contribution 00369 of the LMCE. 


\section{REFERENCES}

Barry R. G. and Chorley R. J. 1987. Atmosphere, weather and climate, ed. Routledge, London and New York: Routledge. 460 .

Brost and Heimann M. 1991. Air pollution modelling and its application (VIII). H. Van Dop Steyn, D.G., Editor. Plenum Press. 465-483.

Ciais P., Tans P. P., White J. W., Trolier M., Francey R., Berry J. A., Randall D., Sellers P. J., Collatz J. G. and Schimel D. S. 1995a. Partitioning of ocean and land uptake of $\mathrm{CO}_{2}$ as inferred by $\mathrm{d} 13 \mathrm{C}$ measurements from the NOAA Climate Monitoring and Diagnostic Laboratory global air sampling network. J. Geophys. Res. 100, 5051-5070.

Ciais, P., Tans, P. P., Trolier, M., White, J. W. C., and Francey, R. 1995b. A large terrestrial $\mathrm{CO}_{2}$ sink indicated by the $\delta^{13} \mathrm{C}$ ratio of atmospheric $\mathrm{CO}_{2}$. Science 269, 1098-1102.

Conway T. J., Tans P. P., Waterman L. S., Thoning K. W., Kitzis D. R., Masarie K. A. and Zhang N. 1994. Evidence for interannual variability of the carbon cycle from the National Oceanic and Atmospheric Administration/Climate Monitoring and Diagnostic Laboratory Global Air Sampling Network. J. Geophys. Res. 99 D11, 22831-22855.

Denning A. S., Fung I. Y. and Randall D. A. 1995. Latitudinal gradient of atmospheric $\mathrm{CO}_{2}$ due to seasonal exchange with land biota. Nature 376, 240-243.

Enting I. G. and Mansbridge J. V. 1989. Seasonal sources and sinks of atmospheric $\mathrm{CO}_{2}$ : Direct inversion of filtered data. Tellus 41B, 111-126.

Enting I. G. and Mansbridge J. V. 1991. Latitudinal distribution of sources and sinks of $\mathrm{CO}_{2}$ : results of an inversion study. Tellus 43B, 156-170.

Enting I. G., Trudinger C. M., Francey R. J. and Granek H. 1993. Synthesis inversion of atmospheric $\mathrm{CO}_{2}$ using the GISS tracer transport model. CSIRO Aust. Div. Atmos. Res. Tech. Pap. 29, 1-44.

Enting I. G., Trudinger C. M. and Francey R. J. 1995. A synthesis inversion of the concentration and $\delta^{13} \mathrm{C}$ of atmospheric $\mathrm{CO}_{2}$. Tellus 47B, 35-51.

Francey R. J., Tans P. P., Allison C. E., Enting I. G., White J. W. C. and Trolier M. 1995. Changes in the oceanic and terrestrial carbon uptake since 1982. Nature 373, 326-329.

Heimann M., 1995. The global atmospheric tracer model TM2: model description and user manual. Max Planck Institut fur Meteorologie, Bundesstrasse 55, D-20146 Hamburg, Germany. Report no. 10.

Heimann M. and Keeling C. D. 1989. A threedimentional model of atmospheric $\mathrm{CO} 2$ transport based on observed winds (2) Model description and simulated tracer experiments. Aspects of climate variability in the Pacific and the Western Americas, Geophysical Monograph 55, Washington (USA).

Heimann M., Keeling C. D. and Tucker C. J. 1989. A three-dimensional model of atmospheric $\mathrm{CO} 2$ trans- port based on observed winds: 3. Seasonal cycle and synoptic time scale variations. Aspects of climate variability in the Pacific and the Western Americas, Geophysical Monograph 55, Washington (USA).

IPCC, 1995. Climate change 1995. Radiative forcing of climate change. An evaluation of the IPCC IS92 emissions scenarios. Ed. IPCC. Cambridge University Press.

Jacob D. J., Prather M. J., Wofsy S. C. and McElroy M. B. 1987. Atmospheric distribution of Kr-85 simulated with a General Circulation Model. J. Geophys. Res. 92 D6, 6614-6626.

Keeling C. D. 1995. Iterannual extremes in the rate of rise of atmospheric carcone dioxide since 1980. Nature 375, 666-670.

Keeling C. D., Bacastow R. B., Carter A. F., Piper S. C., Whorf T. P., Heimann M., Mook W. G. and Roeloffzen H. A. 1989. A three-dimensional model of atmospheric $\mathrm{CO}_{2}$ transport based on observed winds (1) Analysis of observational data. Aspects of Climate Variability in the Pacific and Western Americas, Geophysical Monograph 55. Washington (USA).

Law R., Simmonds I. and Budd W. F. 1992. Appplication of an atmospheric tracer model to the high southern latitudes. Tellus 44B, 358-370.

Liu S. C., McAfee J. R. and Cicerone R. J. 1984. Radon-222 and tropospheric vertical transport. J. Geophys. Res. 89, 7291-7297.

Louis J. F. 1979. A parametric model of vertical eddy fluxes in the atmosphere. Boundary Layer Meteorology 17, 187-202.

Mahlman, J. D. \& Moxim, W. J. 1978. Tracer simulation using a global general circulation model: results from a midlatitude instantaneous source experiment. $J$. of Atmos. Sci 35, 1340-1374.

Mahowald, N. M., Rasch P. J. and Prinn R. G. 1995. Cumulus parametrizations in chemical transport models. J. Geophys. Res. 100, 26173-26189.

Marland G., Rotty R. M. and Treat N. L. $1985 . \mathrm{CO}_{2}$ from fossil fuel burning: global distribution of emissions. Tellus 37, 243-258.

Masarie K. A. and Tans P. P. 1995. Extension and Integration of Atmospheric Carbon Dioxide data into a globally consistent measurement record. J. Geophys. Res. 100 D6, 11593-11610.

Plumb R. A. and Mahlman J. D. 1987. The zonally averaged transport characteristics of the GFDL general circulation/transport model. J. Atmos. Sci. 44, 298-327.

Prather M., McElroy M., Wofsy S., Russell G. and Rind D. 1987. Chemistry of global troposphere: fluorocarbons as tracers of air motion. J. Geophys. Res. 92 D6, 6579-6613.

Ramonet M., 1994. Modélisation du transport atmospherique du dioxyde de carbone dans l'hémisphèere sud. 
Thesis, University of Paris VII, 4 Place Jussieu, 75006 Paris, France.

Ramonet M. Le Roulley, J.C., Bousquet, P., Monfray, P. 1996. Radon-222 measurements during the TropozII campaign and comparison with a global atmospheric transport model. J. Atmos. Chem., 23, 107-136.

Rayner P. J. and Law R. M. 1995, A comparison of modelled responses to prescribed $\mathrm{CO}_{2}$ sources. CSIRO Aust. Div. Atmos. Res. Tech. Pap. no 36, 1-82.

Ruimy A., Saugier B. and Dedieu G. 1994. Methodology for the estimation of terrestrial net primary production from remotely sensed data. J. Geophys. Res. 99, 5263-5283.

Russel G. L. and Lerner J. A. 1981. A new finitedifferencing scheme for the tracer transport equation. J. Appl. Meteor. 20, 1483-1498.
Tans P. P., Conway T. J. and Nakazawa T. 1989. Latitudinal distribution of sources and sinks of atmospheric carbon dioxide derived from surface observations and an atmospheric transport model. J. Geophys. Res. 94 D4, 5151-5172.

Tans P. P., Fung I. Y. and Takahashi T. 1990. Observational Constraints on the Global Atmospheric $\mathrm{CO}_{2}$ budget. Science 247, 1431-1438.

Thoning K. W., Tans P. P. and Komhyr W. D. 1989. Atmospheric carbon dioxide at Mauna Loa Observatory. 2. Analysis of the NOAA GMCC data, 1974,1985. J. Geophys. Res. 94, 8549-8565.

Tiedtke M. 1989. A comprehensive flux scheme for cumulus parametrization in large-scale models. Mont. Weath. Rev. 117, 1779-1800. 